\title{
Detecting nonunitary multiorbital superconductivity with Dirac points at finite energies
}

\author{
J. L. Lado $\circledast^{1,2}$ and M. Sigrist ${ }^{2}$ \\ ${ }^{1}$ Department of Applied Physics, Aalto University, Espoo, Finland \\ ${ }^{2}$ Institute for Theoretical Physics, ETH Zurich, 8093 Zurich, Switzerland
}

(Received 17 August 2019; revised manuscript received 18 October 2019; published 18 November 2019)

\begin{abstract}
Determining the symmetry of the order parameter of unconventional superconductors remains a recurrent topic and nontrivial task in the field of strongly correlated electron systems. Here we show that the behavior of Dirac points away from the Fermi energy is a potential tool to unveil the orbital structure of a superconducting state. In particular, we show that gap openings in such Dirac crossings are a signature of nonunitary multiorbital superconducting order. Consequently, also spectral features at higher energy can help us to identify broken symmetries of superconducting phases and the orbital structure of nonunitary states. Our results show how angle-resolved photoemission spectroscopy measurements can be used to detect nonunitary multiorbital superconductivity.
\end{abstract}

DOI: 10.1103/PhysRevResearch.1.033107

\section{INTRODUCTION}

Superconductors belong to the most intriguing states of matter, through their rich phenomenology, the range of underlying physical mechanisms, their different solid-state realizations, and, ultimately, their potential technological applications. Nowadays we label superconductors as conventional or unconventional depending on the symmetry properties of their Cooper-pairing states. Conventional includes all superconductors realizing Cooper pairs of highest symmetry, whose wave function is essentially structureless and spin singlet. In contrast, the term unconventional encompasses all other cases, in particular, those with superconducting order parameters which violate additional symmetries such as lattice, timereversal, or orbital symmetries, or form topologically nontrivial phases [1-3]. Strongly correlated electron systems provide an especially fertile ground for the unconventional superconductivity as they offer a large variety of low-energy fluctuations to favoring different Cooper-pairing channels [4,5].

While the major concepts for unconventional superconductivity are based on single-band descriptions, multiorbital features increase complexity. For many of the recently found superconductors, a multiorbital approach seems mandatory to understand the intricate relation of the superconducting phase with the orbital degrees of freedom and to yield sensible mechanisms for pairing [6,7]. Very recent experiments on Fechalcogenides [7] may show some intriguing aspect of orbital selectivity of the superconducting phase [7]. In angle-resolved photoemission spectroscopy (ARPES), a conspicuous gap opening of Dirac points rather far away from the Fermi energy has been observed and interpreted in terms of spontaneous

Published by the American Physical Society under the terms of the Creative Commons Attribution 4.0 International license. Further distribution of this work must maintain attribution to the author(s) and the published article's title, journal citation, and DOI. time-reversal symmetry breaking stemming from a spontaneous magnetization associated to the superconducting state $[8,9]$. Motivated by this finding, we would like to address this feature of the remote Dirac points [10] from a general viewpoint and show that this peculiarity may give interesting insights into the multiorbital nature of the superconducting phase on a general setup.

Here, we demonstrate that a gap opening in a remote Dirac point upon entering a superconducting state indicates the realization of nonunitarity of Cooper-pairing states in orbital space, due to specific forms of orbital selective pairing. The basic phenomenology is depicted in Fig. 1, showing in Fig. 1(a) the normal-state spectrum with a Dirac point remote from the Fermi level. After extending the electronic states by holes to the Nambu space, we obtain the standard spectrum of a superconductor with a gap around the Fermi energy [Figs. 1(b) and 1(c)]. A unitary pairing state leaves the Dirac points untouched [Fig. 1(b)], while for nonunitary phases these Dirac points disappear by a gap opening [Fig. 1(c)]. In the enlarged Fig. 1(d) we indicate that the size of the gap is controlled by $\Upsilon$, a measure for nonunitarity, as we define below. We note that the physical mechanism leading to the superconducting state can be of various kinds, including charge or antiferromagnetic fluctuation, yet its nature will not affect our analysis. The paper is organized as follows: In Sec. II we show an analysis based on a minimal Dirac equation, in Sec. III we show how our mechanism applies to a real-space model, in Sec. IV we present how the nonunitarity creates topological interface excitations, and in Sec. V we summarize our conclusions.

\section{NONUNITARITY IN A CONTINUUM MODEL}

For the theoretical approach to this problem, we first consider a generic two-orbital system with a two-dimensional Bloch Hamiltonian hosting a Dirac crossing [11] away from the Fermi level [Fig. 1(a)]. We focus on the region near the Dirac points of a two-dimensional band structure using a $\mathbf{k} \cdot \mathbf{p}$ description with the spinor basis $\psi_{\mathbf{k}}^{\dagger}=\left(c_{\alpha, \mathbf{k}}^{\dagger}, c_{\beta, \mathbf{k}}^{\dagger}\right)$, where 

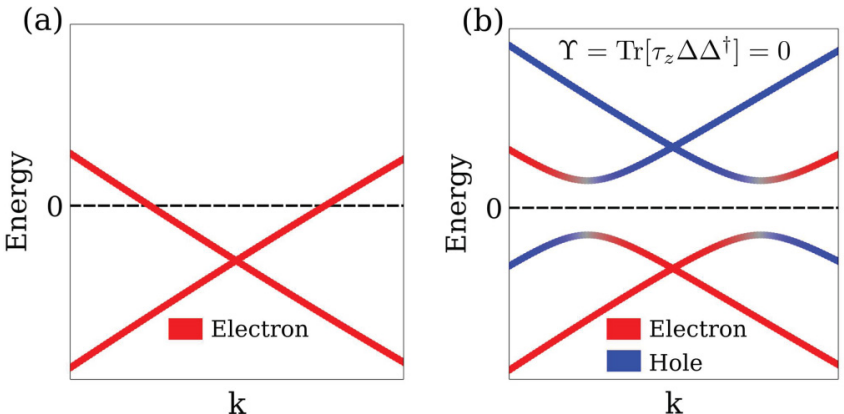

$\mathrm{k}$
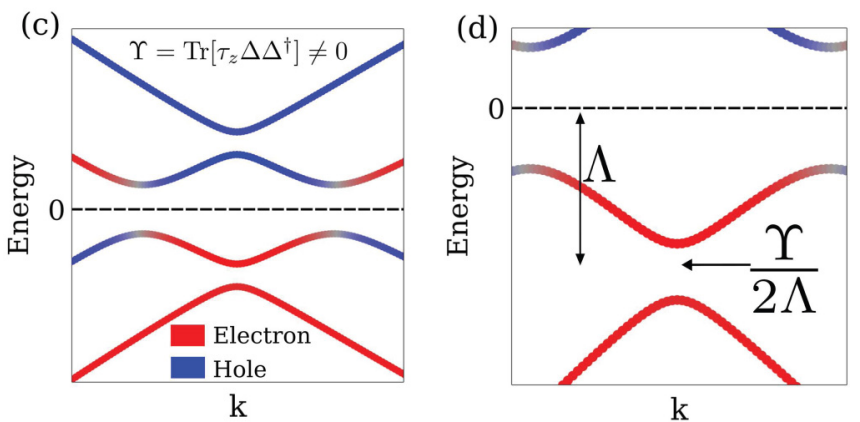

$\mathrm{k}$

FIG. 1. (a) Sketch of the normal-state band structure, featuring a Dirac crossing away from the chemical potential. (b) Sketch of the band structure in a unitary superconducting state, showing that the Dirac point stays closed. In stark comparison, the onsite of a nonunitary superconducting state opens up the Dirac point away from the chemical potential as shown in (c). The magnitude of the gap opening at the Dirac crossing depends on the nonunitarity $\Upsilon$ and the distance to the chemical potential as shown in (d).

$c_{\alpha, \mathbf{k}}^{\dagger}$ and $c_{\beta, \mathbf{k}}^{\dagger}$ denote electronic creation operators of Bloch states with momentum $\mathbf{k}$ and the indices $\alpha, \beta$ label the two orbitals, including also the spin degrees of freedom. Then, the effective Hamiltonian is written as $\mathcal{H}_{0}^{D P}(\mathbf{k})=\psi_{\mathbf{k}}^{\dagger} H_{0}^{D P}(\mathbf{k}) \psi_{\mathbf{k}}$, where $H_{0}^{D P}(\mathbf{k})$ is a $2 \times 2$ matrix of the form

$$
H_{0}^{D P}(\mathbf{k})=-\Lambda \tau_{0}+\tau_{x} k_{x}+\tau_{y} k_{y} .
$$

The absence of the Pauli matrix $\tau_{z}$ in Eq. (1) guarantees the existence of a Dirac crossing at the energy $-\Lambda$.

We now turn to the superconducting state. For simplicity, we consider here pairing of electrons between timereversal Kramers partners, which includes conventional superconducting states. Note that other channels such as spin-triplet pairing states could be treated in an analogous way. Thus, we extend the Hamiltonian to $\mathcal{H}_{\mathrm{BdG}}(\mathbf{k})=$ $\mathcal{H}_{0}^{D P}(\mathbf{k})+\frac{1}{2} \sum_{i, j \in \alpha, \beta} c_{i, \mathbf{k}}^{\dagger} c_{\bar{j},-\mathbf{k}}^{\dagger} \Delta_{i j}(\mathbf{k})+$ H.c., where $\bar{\alpha}, \bar{\beta}$ label the Kramers time-reversal partners of $\alpha, \beta$ in orbital and spin space. We write the $2 \times 2$ gap matrix as

$$
\Delta(\mathbf{k})=\left(\begin{array}{cc}
\Delta_{\alpha \alpha}(\mathbf{k}) & \Delta_{\alpha \beta}(\mathbf{k}) \\
\Delta_{\beta \alpha}(\mathbf{k}) & \Delta_{\beta \beta}(\mathbf{k})
\end{array}\right)
$$

with $\Delta_{\alpha \alpha}(\mathbf{k})=\left\langle c_{\alpha, \mathbf{k}} c_{\bar{\alpha},-\mathbf{k}}\right\rangle, \Delta_{\alpha \beta}(\mathbf{k})=\left\langle c_{\alpha, \mathbf{k}} c_{\bar{\beta},-\mathbf{k}}\right\rangle, \Delta_{\beta \alpha}(\mathbf{k})=$ $\left\langle c_{\beta, \mathbf{k}} c_{\bar{\alpha},-\mathbf{k}}\right\rangle, \Delta_{\beta \beta}(\mathbf{k})=\left\langle c_{\beta, \mathbf{k}} c_{\bar{\beta},-\mathbf{k}}\right\rangle$.

For systems where $s_{z}$ is a good quantum number, the indices are $\alpha \equiv\left(\alpha_{0}, \uparrow\right)$ and $\bar{\alpha} \equiv\left(\alpha_{0}, \downarrow\right)$ with $\alpha_{0}$ the orbital label, such that $\Delta(\mathbf{k}) \equiv \Delta^{\uparrow \downarrow}(\mathbf{k})$ describes opposite spin pairing. For concreteness, we continue now with this orbital spin labeling, although more general Kramers pairs yield the same behavior. With the Nambu spinor $\Psi_{\mathbf{k}}^{\dagger}=\left(c_{\alpha_{0}, \uparrow, \mathbf{k}}^{\dagger}, c_{\beta_{0}, \uparrow, \mathbf{k}}^{\dagger}, c_{\alpha_{0}, \downarrow,-\mathbf{k}}, c_{\beta_{0}, \downarrow,-\mathbf{k}}\right)$, the Bogoliubov-de Gennes (BdG) Hamiltonian $H_{\mathrm{BdG}}$ takes the form $\mathcal{H}_{\mathrm{BdG}}=$ $\frac{1}{2} \Psi_{\mathbf{k}}^{\dagger} H_{\mathrm{BdG}}(\mathbf{k}) \Psi_{\mathbf{k}}+$ H.c., where $H_{\mathrm{BdG}}(\mathbf{k})$ is the following $4 \times 4$ matrix:

$$
H_{\mathrm{BdG}}(\mathbf{k})=\left(\begin{array}{cc}
H_{0}^{D P}(\mathbf{k}) & \Delta(\mathbf{k}) \\
\Delta(\mathbf{k}) & -H_{0}^{D P}(-\mathbf{k})
\end{array}\right) .
$$

Using this Hamiltonian, we now derive the corrections induced by the Cooper pairing at the Dirac point of Eq. (1). A Schrieffer-Wolff transformation equivalent to second-order perturbation theory leads to the effective Hamiltonian around the Dirac point $\mathbf{k}=\mathbf{K}$ of the form

$$
H^{D P}=H_{0}^{D P}+\frac{\Delta \Delta^{\dagger}}{2 \Lambda}
$$

with $\Delta \equiv \Delta(\mathbf{K})$, valid in the limit $|\Lambda| \gg \max (|\Delta|)$.

As a $2 \times 2$ matrix, the correction to the Dirac Hamiltonian in Eq. (4) can be decomposed into the identity $\mathcal{I}$ and Pauli matrices $\tau_{i}$ as $\frac{\Delta \Delta^{\dagger}}{2 \Lambda}=\gamma_{0} \mathcal{I}+\gamma_{x} \tau_{x}+\gamma_{y} \tau_{y}+\gamma_{z} \tau_{z}$ with $\gamma_{0}, \gamma_{x}, \gamma_{y}, \gamma_{z}$ real numbers, where $\gamma_{x}=\gamma_{y}=\gamma_{z}=0$ defines a unitary pairing state. Generally, this yields an effective Hamiltonian around the original Dirac point that can be again written in terms the identity $\mathcal{I}$ and Pauli matrices $\tau_{i}$ as

$$
H^{D P}=-\bar{\Lambda} \tau_{0}+\bar{p}_{x} \tau_{x}+\bar{p}_{y} \tau_{y}+\gamma_{z} \tau_{z}
$$

with $\bar{\Lambda}=\Lambda-\gamma_{0}, \quad \bar{p}_{x}=p_{x}+\gamma_{x}$, and $\bar{p}_{y}=p_{y}+\gamma_{y}$. This highlights that a nonunitary superconducting term modifies the Dirac crossing away from the chemical potential. As a result, the existence of nonunitary pairing can be generically observed by analyzing the behavior of Dirac point in the superconducting state. For nonvanishing $\gamma_{x} \neq 0$ or $\gamma_{y} \neq 0$, the onset of superconductivity will shift the Dirac point in reciprocal space. But, more importantly, for $\gamma_{z} \neq 0$ in Eq. (5) a gap opens at the Dirac point. Generally, the condition to open a gap at the Dirac point can be written in terms of the nonunitarity parameter $\Upsilon$ of the gap matrix as $\Upsilon=$ $\sum_{\nu=\alpha, \beta}\left[\Delta_{\alpha, \nu} \Delta_{\alpha, \nu}^{*}-\Delta_{\beta, \nu} \Delta_{\beta, \nu}^{*}\right]$, which in matrix form reads as

$$
\Upsilon=\operatorname{Tr}\left[\tau_{z} \Delta \Delta^{\dagger}\right] \neq 0
$$

yielding $\gamma_{z}=\Upsilon / 4 \Lambda$ in Eq. (5). We point out that a similar analysis could be carried out both for one- and threedimensional systems described by a $2 \times 2$ Dirac equation. However, in the three-dimensional case, a gap can not be induced in the $2 \times 2$ Dirac Hamiltonian, as any correction will only create a momentum shift.

We examine now a few examples based on this criterion. We start with the unitary states. First the intraorbital state given by $\Delta(\mathbf{k})=\Delta_{0} \tau_{0} f(\mathbf{k})$ which for $f(\mathbf{k})=$ const corresponds to a conventional superconductor. This leads obviously to $\Upsilon=0$. The same is true of an interorbital pairing state like $\Delta(\mathbf{k})=\Delta_{0} \tau_{x} f(\mathbf{k})$. A linear combination of these two states is nonunitary with $\Upsilon=0$, and leads to a shift of the Dirac points.

A straightforward case opening a gap is the intraorbital pairing state $\Delta(\mathbf{k})=\Delta_{0} f(\mathbf{k})\left(\begin{array}{ll}1 & 0 \\ 0 & 0\end{array}\right)=\Delta_{0} f(\mathbf{k})\left(\tau_{0}+\tau_{z}\right) / 2$, which corresponds to pairing restricted to one orbital only and leads to $\Upsilon \neq 0$. Such a state breaks orbital symmetry, and 
it is expected to appear close to a quantum critical point to an orbital ordered instability. An exemplary nonunitary state for interorbital pairing is $\Delta=\Delta_{0} f(\mathbf{k})\left(\begin{array}{ll}0 & 1 \\ 0 & 0\end{array}\right)=\Delta_{0} f(\mathbf{k})\left(\tau_{x}+\right.$ $\left.i \tau_{y}\right) / 2$, which shows spontaneous time-reversal symmetry breaking in orbital space and realizes an antiferromagnetic superconducting state breaking spin-rotational symmetry. This state is expected to appear close to a quantum phase transition to an antiferromagnetic instability. We finally emphasize that in the previous description we have not assumed any momentum-space structure of the superconducting state, and as a result this argument can be applied to arbitrary momentum superconducting structures.

\section{NONUNITARITY IN A REAL-SPACE MODEL}

We now illustrate this analysis on a specific tight-binding model for the honeycomb lattice. The role of orbitals is now played by the two sublattices. The Hamiltonian has the form

$$
\begin{aligned}
\mathcal{H}_{T B}= & t \sum_{\langle i j\rangle, s} c_{i, s}^{\dagger} c_{j, s}+\Lambda_{1} \sum_{i, s} c_{i, s}^{\dagger} c_{i, s} \\
& +\Lambda_{2} \frac{i}{3 \sqrt{3}} \sum_{\langle\langle i j\rangle\rangle, s, s^{\prime}} v_{i j} \sigma_{z}^{s s^{\prime}} \tau_{z}^{i j} c_{i, s}^{\dagger} c_{j, s^{\prime}} \\
& +\sum_{i}\left(\Delta_{i}^{\uparrow \downarrow} c_{i, \uparrow} c_{i, \downarrow}+\text { H.c. }\right)
\end{aligned}
$$

with $c_{i s}^{\dagger}, c_{i s}$ the creation and annihilation operators of electrons on the site $i$ with spin $s,\langle\ldots\rangle$ denotes the summation over nearest neighbors, $\langle\langle\ldots\rangle\rangle$ over next-nearest neighbors, $\sigma^{z}$ is the corresponding spin Pauli matrix, and $v_{i j}= \pm 1$ for clockwise or anticlockwise hopping within each hexagonal plaquette, whereby an additional sign difference enters through the orbital Pauli matrix $\tau_{z}$, giving the opposite sign for the two sublattices. Note that the $\Lambda_{2}$ term describes the noncentrosymmetric spin-orbit coupling for next-nearest-neighbor hopping, which induces a momentum-dependent spin splitting in the band structure with shifted Dirac cones [12,13]. The $\Lambda_{1}$ term introduces merely the chemical potential which is finite and negative for the electron-doped system. Finally, $\Delta_{i}^{\uparrow \downarrow}$ controls the amplitude of the onsite pairing state, yielding an intraorbital pairing. We will use here the simplest spin-singlet $s$-wave superconducting pairing to highlight the impact of a nontrivial orbital structure even for a topologically trivial superconducting state. Other superconducting momentum structures could be studied analogously $[14,15]$.

The Hamiltonian (7) has two simple limiting cases. First, for $\Lambda_{1} \neq 0$ and $\Lambda_{2}=0$, the band structure shows two Dirac points below the Fermi level, realizing a doped honeycomb lattice [Fig. 2(a)]. Second, with $\Lambda_{1}=0$ and $\Lambda_{2} \neq 0$ the system is half-filled, but the Dirac cones are spin split due to the noncentrosymmetric spin-orbit coupling, and there are again Dirac points away from the Fermi energy [Fig. 2(b)]. In the generic case, $\Lambda_{1} \neq 0$ and $\Lambda_{2} \neq 0$, Dirac crossings will appear at energies $\Lambda=\Lambda_{1} \pm \Lambda_{2}$.

Now, we introduce the nonunitary pairing state $\Delta=$ $\Delta_{0}\left(\tau_{0}+\tau_{z}\right) / 2$, which corresponds to onsite pairing on one of the two sublattices only. For both limiting cases, the Dirac point remote from the Fermi energy develops a gap as seen
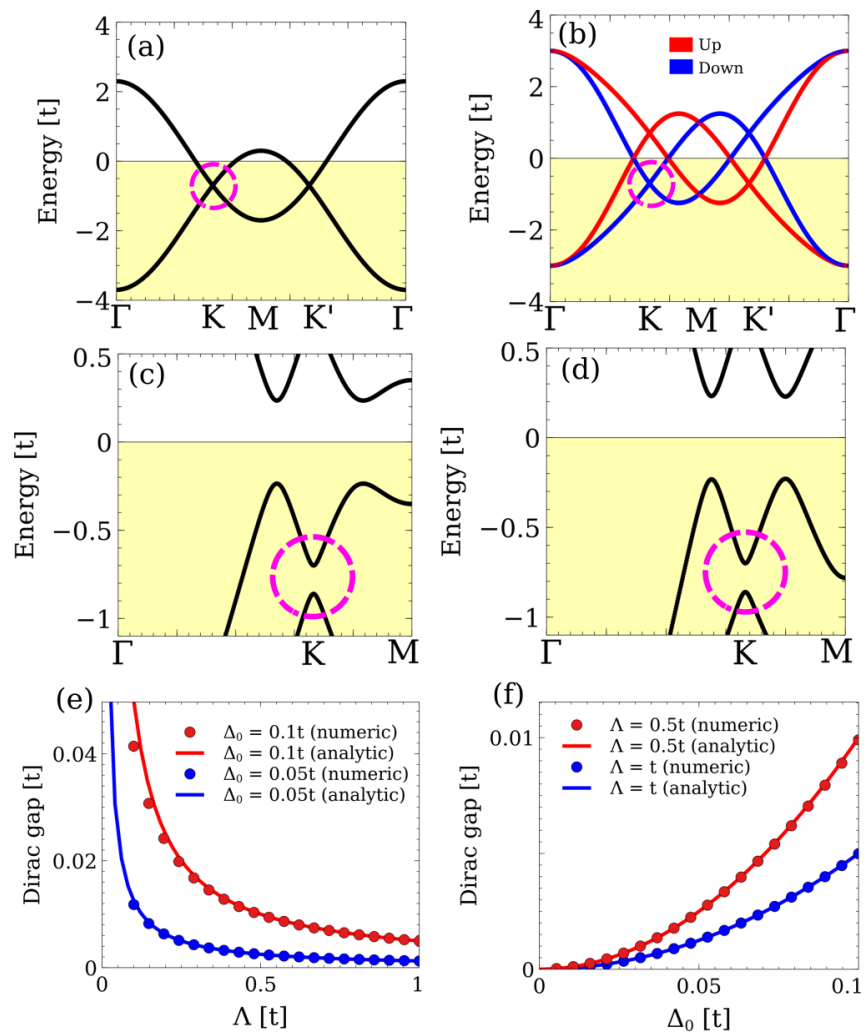

FIG. 2. Normal-state band structure (a), (b) for a Dirac band system in the normal state, showing Dirac crossings away from the chemical potential. (a) Shows a doped honeycomb lattice without spin-orbit coupling, whereas (b) shows a half-filled honeycomb system with noncentrosymmetric spin-orbit coupling (b). (c) Shows the BdG spectra in the presence of nonunitary superconducting term for (a), and (d) the analogous spectra for the case (b). In both scenarios, it is observed that the nonunitary superconducting state drives a gap opening at the Dirac point. (e), (f) Show a comparison between the numeric and analytic results for Dirac gap opening. In particular, (e) shows the Dirac gap as a function of the distance to the chemical potential $\Lambda$ (e), and (f) the Dirac gap as a function of the nonunitary superconducting term. We took $\Lambda_{1}=0.7 t$ and $\Lambda_{2}=0$ for (a), (c), $\Lambda_{1}=0.0$ and $\Lambda_{2}=0.7 t$ for (b), (d), $\Lambda_{2}=0$ for panels (e), (f), and $\Delta_{0}=0.71 \Lambda$ for (c), (d).

in Figs. 2(c) and 2(d). Moreover, we compute the magnitude of this gap as function of $\Lambda=\Lambda_{1}$ and $\Delta_{0}$ and compare this with our result for $\Upsilon / 2 \Lambda$ of Eq. (6) demonstrating very good agreement as long as the condition $|\Lambda| \gg\left|\Delta_{0}\right|$ is satisfied [Figs. 2(e) and 2(f)]. Note that this pairing state is a so-called pair density wave state and corresponds to a spontaneous sublattice symmetry breaking. It would be accompanied by a charge density difference on the two sublattices and is stabilized in systems where superconductivity appears close to quantum phase transition to a charge density wave instability.

We now discuss the electron spectra function which is experimentally accessible, for instance, by ARPES. This can be computed through

$$
A(\omega, \mathbf{k})=-\frac{1}{\pi} \operatorname{Im}\left(\operatorname{Tr}\left\{P_{e}\left[\omega-H(\mathbf{k})+i 0^{+}\right]^{-1}\right\}\right)
$$


(a)

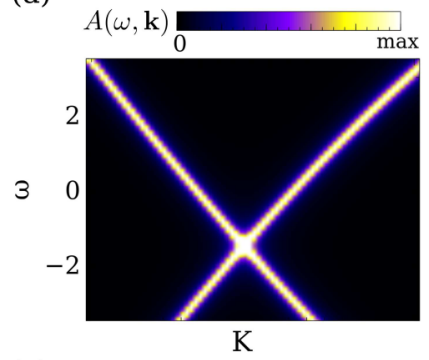

(c)

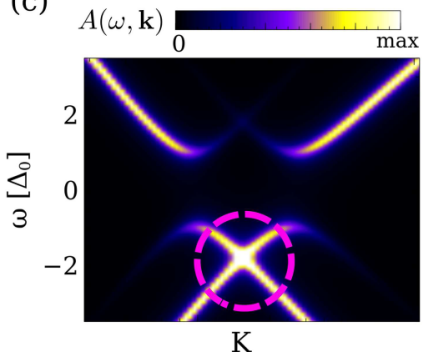

(e)

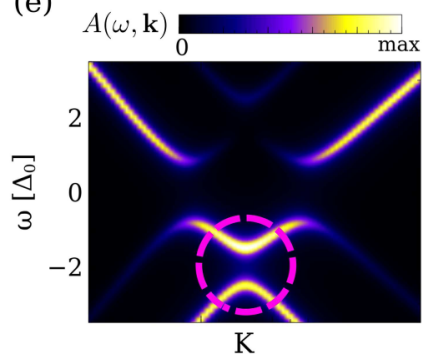

(b)

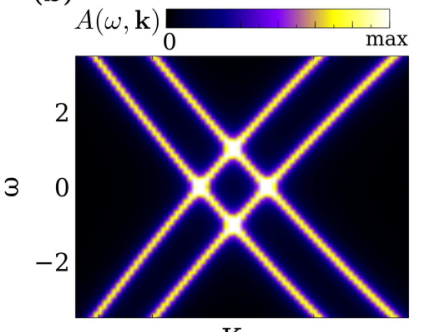

K

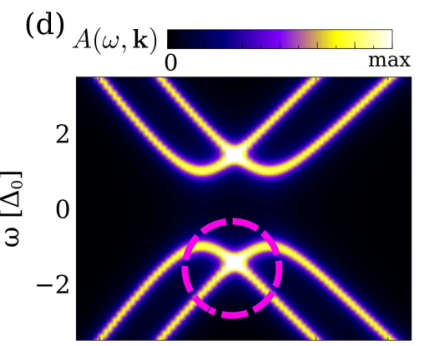

K

(f)

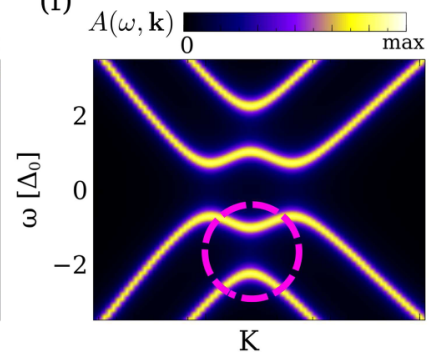

FIG. 3. Electron spectral function $A(\omega, \mathbf{k})$ for a system with a Dirac point away from the chemical potential due to doping (a), (c), (e) and due to spin-orbit coupling (b), (d), (f). (a), (b) Show the spectral function in the normal state, depicting a Dirac crossing away form the chemical potential. In the presence of unitary orbital superconductivity, a gap opens up at the chemical potential, but not at the Dirac crossings (c), (d). In contrast, in the presence of a nonunitary superconducting state, a gap opens up at the Dirac crossing (e), (f). We took $\Lambda_{1}=1.5 \Delta_{0}$ and $\Lambda_{2}=0$ in (a), (c), (e), $\Lambda_{1}=0$ and $\Lambda_{2}=\Delta_{0}$ in (b), (d), (f).

with $H(\mathbf{k})$ as the corresponding $\mathrm{BdG}$ Hamiltonian in the Nambu representation and $P_{e}$ the projection operator on the electron sector. The quantity $A(\omega, \mathbf{k})$ would be the observable in an ARPES experiment in the superconducting state, as shown in Fig. 3. We consider again the two limiting cases for the Dirac cones shifted below the Fermi energy in Figs. 3(a), 3(c), and 3(e) for electron doping and in Figs. 3(b), 3(d), and 3(f) for spin-orbit coupling. The spectrum for the normal-state spectrum is shown in Figs. 3(a) and 3(b), and for superconducting states in Figs. 3(c)-3(f), both for a unitary [Figs. 3(c) and 3(d)] and a nonunitary [Figs. 3(e) and 3(f)] superconducting state. The unitary state is given by $\Delta^{U}=\Delta_{0} \tau_{0}$ and the nonunitary one by $\Delta=\Delta_{0}\left(\tau_{0}+\tau_{z}\right) / 2$. As anticipated from our discussion above, again a gap opens at the Dirac points below from the Fermi level only if the pairing state is nonunitary. In all cases, the superconducting gap at the Fermi energy is fully open.

The real-space analysis demonstrates that the mechanism we presented is robust against corrections to the Dirac dispersion such as trigonal warping or electron-hole asymmetry,

(a)

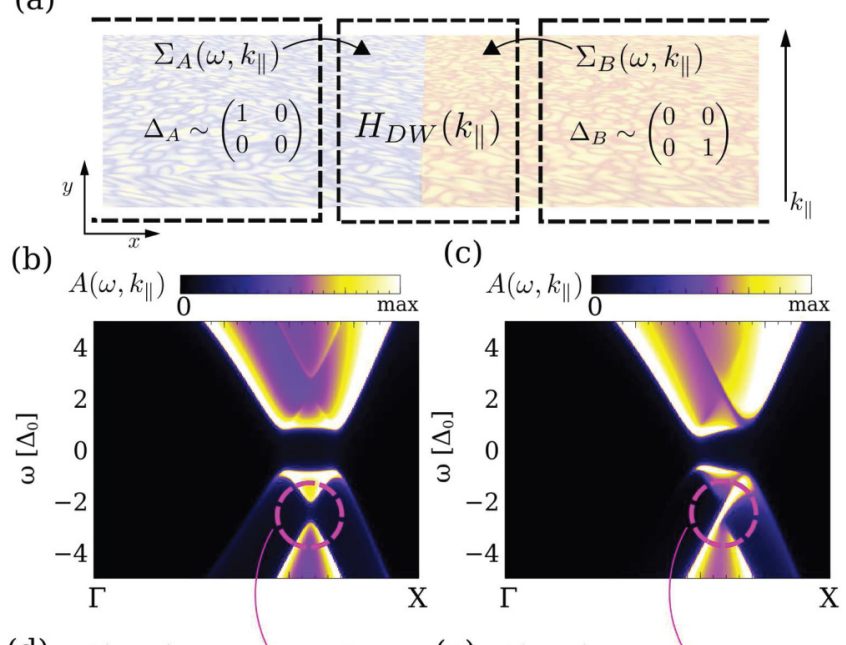

(d)

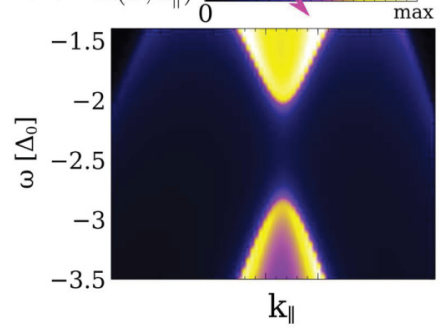

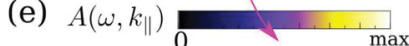

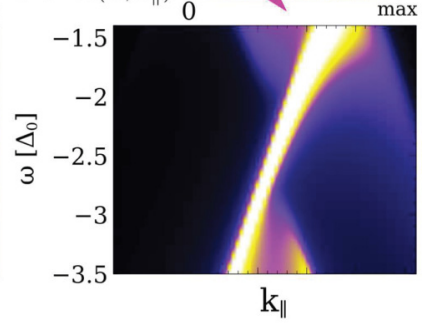

FIG. 4. (a) Sketch of an interface between two superconducting domains with different nonunitary orders $\Delta_{A}$ and $\Delta_{B}$. (b), (c) Show the bulk spectral function of the superconductor in the absence of a superconducting domain wall, showing the gap opening in the buried Dirac point (b) and (d). In contrast, at the interface between the two superconducting domains, a chiral excitation appears inside the Dirac opening as shown in (c) and (e). We took $\Lambda=1.5 \Delta_{0}$.

present in the lattice model but not in the continuum model. As a result, the lattice model demonstrates that our phenomenology is a robust mechanism that will apply even in cases beyond a minimal Dirac equation, highlighting that it will be applicable to dispersions of real compounds. This will be especially important in the next section, when we show the emergence of nontrivial interface excitation on a real-space boundary.

\section{INTERFACE MODES ASSOCIATED TO NONUNITARITY}

Gapped Dirac points are a known source of topological excitations. In the following, we show how these gaps give rise to in-gap excitations at domain walls between superconducting domains, which otherwise lack any spectroscopic signature at the Fermi energy. For this purpose, we now take an interface between two degenerate nonunitary superconducting phases $A$ and $B$, namely, $\Delta_{A}=\Delta_{0}\left(\tau_{0}+\tau_{z}\right) / 2$ and $\Delta_{B}=\Delta_{0}\left(\tau_{0}-\tau_{z}\right) / 2$. We now consider a system with translational invariance along the domain wall, direction $\|$, with $\Delta_{A}$ on the left and $\Delta_{B}$ on the right [see Fig. 4(a)]. Here, the spectral function at the superconducting domain wall can be obtained by the Green's function $G\left(\omega, k_{\|}\right)=$ $\left[\omega-H_{D W}\left(k_{\|}\right)-\Sigma_{A}\left(\omega, k_{\|}\right)-\Sigma_{B}\left(\omega, k_{\|}\right)\right]^{-1}$ where $k_{\|}$is the 
momentum parallel to the domain wall, and $H_{D W}$ denotes the local Hamiltonian at the interface, where, for simplicity, we take a sharp transition between the two superconducting gaps. The self-energies $\Sigma_{A, B}\left(\omega, k_{\|}\right)$are induced by the semiinfinite superconducting regions, which can be exactly computed through a frequency-dependent renormalization group algorithm [16]. Then, the spectral function at the interface can be computed by $A\left(\omega, k_{\|}\right)=-\frac{1}{\pi} \operatorname{Im}\left\{\operatorname{Tr}\left[P_{e} G\left(\omega, k_{\|}\right)\right]\right\}$, which gives access to the local excitations. Physically, the system we are computing corresponds to an interface between two semi-infinite superconducting orders with a sharp transition between, yet this procedure can be trivially extended to a smooth transition.

Away from the domain wall, the Dirac point shows a gap in the k-resolved spectral function [Figs. 4(b) and 4(d)]. At the domain wall, a chiral state appears inside the gap, corresponding to a chiral Andreev bound state [Figs. 4(c) and $4(\mathrm{e})$ ]. The origin of such in-gap excitations can be easily rationalized by defining the Chern number associated to the effective Dirac Hamiltonian (4):

$$
C=\frac{1}{2} \operatorname{sign}(\Upsilon) \operatorname{sign}(\Lambda) \text {. }
$$

Taking $\Delta_{0}>0$ for $\Delta_{A}$ and $\Delta_{B}$, we obtain $C_{A}=+1 / 2$ and $C_{B}=-1 / 2$, which from the index theorem implies that a single interface state will appear inside the Dirac gap [Fig. 4(e)] [17]. We emphasize that the noninteger nature of the Chern number stems from the fact that the Dirac equation is defined in a noncompact manifold, which is associated with taking a Dirac equation as a low-energy approximation. Interestingly, such interface states in the Dirac gap do not create an Andreev bound state within the actual superconducting gap at the Fermi level. This shows that such domain walls are invisible in the actual low-energy spectroscopic gap of the superconductor, but can be observed by targeting Dirac point openings away from the chemical potential. Note that there are no states inside the Dirac opening in a boundary with vacuum, ${ }^{1}$ such

\footnotetext{
${ }^{1}$ A zigzag interface with vacuum will show a flat band of zigzag boundary modes, yet without crossing the gap.
}

that only the gap opening at the Dirac points would be visible in ARPES at a surface. We finally mention that the real-space model allows to clearly demonstrate that emergence of interface excitations between the two superconducting domains by exactly computing such a real-space interface. This robustly shows that potential corrections to the low-energy model do not spoil the topological interface modes, demonstrating that an approximate analysis in terms of a low-energy Dirac equation can yield qualitatively faithful results.

\section{CONCLUSIONS}

To summarize, we have shown that a gap opening at a Dirac point away from the Fermi level is a hallmark of a multiorbital nonunitary state. In particular, our results show that information on the orbital symmetry of the superconducting state could be directly extracted from an ARPES experiment, providing a simple spectroscopic technique to detect unconventional nonunitary superconductivity of this kind. On the one hand, our methodology can be readily applied to iron chalcogenides, where signatures of orbitalselective states have been observed [7], and that are known to host remote Dirac crossings in the band structure [18]. On the other hand, our results may potentially provide an alternative route to characterize the superconducting state of twisted bilayer graphene [19-21], as it hosts Dirac points $10 \mathrm{meV}$ below the Fermi level [22-25]. Ultimately, our results highlight that ARPES measurements can help to identify the origin of superconductivity in Dirac compounds. Moreover, it may also reveal the presence of an incipient order, such as a charge density wave in our example above, which might be involved in the pairing mechanism due to strong fluctuations.

\section{ACKNOWLEDGMENTS}

We are grateful to P. Johnson for many helpful discussions on his experimental results. J.L.L. is grateful for financial support from the ETH Fellowship program and M.S. from the Swiss National Science Foundation through Division II (Grant No. 184739).
[1] A. J. Leggett, Rev. Mod. Phys. 47, 331 (1975).

[2] M. Sigrist and K. Ueda, Rev. Mod. Phys. 63, 239 (1991).

[3] M. Sato and Y. Ando, Rep. Prog. Phys. 80, 076501 (2017).

[4] C. Pfleiderer, Rev. Mod. Phys. 81, 1551 (2009).

[5] D. J. Scalapino, Rev. Mod. Phys. 84, 1383 (2012).

[6] G. R. Stewart, Rev. Mod. Phys. 83, 1589 (2011).

[7] P. O. Sprau, A. Kostin, A. Kreisel, A. E. Böhmer, V. Taufour, P. C. Canfield, S. Mukherjee, P. J. Hirschfeld, B. M. Andersen, and J. C. S. Davis, Science 357, 75 (2017).

[8] N. Zaki, G. Gu, A. M. Tsvelik, C. Wu, and P. D. Johnson, arXiv:1907.11602.

[9] L.-H. Hu, P. D. Johnson, and C. Wu, arXiv:1906.01754.

[10] L. Komendová, A. V. Balatsky, and A. M. Black-Schaffer, Phys. Rev. B 92, 094517 (2015).

[11] T. Wehling, A. Black-Schaffer, and A. Balatsky, Adv. Phys. 63, 1 (2014).
[12] F. D. M. Haldane, Phys. Rev. Lett. 61, 2015 (1988).

[13] C. L. Kane and E. J. Mele, Phys. Rev. Lett. 95, 226801 (2005).

[14] P. M. R. Brydon, D. S. L. Abergel, D. F. Agterberg, and V. M. Yakovenko, Phys. Rev. X 9, 031025 (2019).

[15] P. M. R. Brydon, D. F. Agterberg, H. Menke, and C. Timm, Phys. Rev. B 98, 224509 (2018).

[16] M. P. L. Sancho, J. M. L. Sancho, J. M. L. Sancho, and J. Rubio, J. Phys. F: Met. Phys. 15, 851 (1985).

[17] R. Jackiw and C. Rebbi, Phys. Rev. D 13, 3398 (1976).

[18] S. Y. Tan, Y. Fang, D. H. Xie, W. Feng, C. H. P. Wen, Q. Song, Q. Y. Chen, W. Zhang, Y. Zhang, L. Z. Luo, B. P. Xie, X. C. Lai, and D. L. Feng, Phys. Rev. B 93, 104513 (2016).

[19] Y. Cao, V. Fatemi, S. Fang, K. Watanabe, T. Taniguchi, E. Kaxiras, and P. Jarillo-Herrero, Nature 556, 43 (2018). 
[20] M. Yankowitz, S. Chen, H. Polshyn, Y. Zhang, K. Watanabe, T. Taniguchi, D. Graf, A. F. Young, and C. R. Dean, Science 363, 1059 (2019).

[21] X. Lu, P. Stepanov, W. Yang, M. Xie, M. A. Aamir, I. Das, C. Urgell, K. Watanabe, T. Taniguchi, G. Zhang, A. Bachtold, A. H. MacDonald, and D. K. Efetov, Nature 574, 653 (2019).

[22] E. Suárez Morell, J. D. Correa, P. Vargas, M. Pacheco, and Z. Barticevic, Phys. Rev. B 82, 121407(R) (2010).
[23] J. M. B. Lopes dos Santos, N. M. R. Peres, and A. H. Castro Neto, Phys. Rev. Lett. 99, 256802 (2007).

[24] R. Bistritzer and A. H. MacDonald, Proc. Natl. Acad. Sci. USA 108, 12233 (2011).

[25] S. L. Tomarken, Y. Cao, A. Demir, K. Watanabe, T. Taniguchi, P. Jarillo-Herrero, and R. C. Ashoori, Phys. Rev. Lett. 123, 046601 (2019). 